1. By means of a certain trigonometric sum of order $n$ at most, with an error not exceeding

$$
\frac{1}{n}\left(c_{1} \lambda+c_{2} \frac{\nu}{\delta}\right)
$$

where $c_{1}$ and $c_{2}$ (like $c_{3}, \cdots, c_{6}$ below) are absolute constants, and $\nu$ is the difference between the upper and lower limits of $f(x)$.

2. By means of Fejér's arithmetic mean of the first $n+1$ terms $(n>1)$ of the Fourier series of $f(x)$, with an error not exceeding

$$
\frac{\log n}{n}\left(c_{3} \lambda+c_{4} \frac{\nu}{\delta}\right) \text {. }
$$

3. By means of the first $n+1$ terms $(n>1)$ of the Fourier series itself, with an error not exceeding

$$
\frac{\log n}{n}\left(c_{5} \lambda+c_{6} \mu \frac{\nu}{\delta}\right)
$$

where $\mu$ is the number of discontinuities in any interval of length $2 \pi$.

18. The systems of curves studied by Professor Kasner play (roughly) the same role in the geometry of the dual variable $u+j v\left(j^{2}=0\right)$ as the isothermal systems in the geometry of the ordinary complex variable $x+i y\left(i^{2}=-1\right)$. The analogy is not complete, since the Laplace equation $\psi_{x x}+\psi_{y y}=0$ is replaced by the simpler equation $\psi_{v v}=0$, but a list of analogous properties (including new results for the isothermal type) is obtained.

F. N. Cole, Secretary.

\title{
THREE OR MORE RATIONAL CURVES COLLINEARLY RELATED.
}

BY DR. JOSEPH E. ROWE.

(Read before the American Mathematical Society, December 31, 1912.)

\section{Introduction.}

The $R^{n}$, or rational plane curve of order $n$, possesses certain sets* of covariant rational point and line curves which

* J. E. Rowe, "Bicombinants of the rational plane quartic and combinant curves of the rational plane quintic," Transactions, vol. 13 (July, 1912), pp. 388-389. 
are related in the following manner: if $A, B$, and $C$ are three rational point loci of such a set, any parameter value substituted in the parametric equations of these curves yields the coordinates of three collinear points; similarly, if $A^{\prime}, B^{\prime}$, and $C^{\prime}$ are three line loci of such a set, any parameter value substituted in their parametric equations yields the coordinates of three concurrent lines. Curves related in either of the above ways may be called collinearly related, or curves in collinear relation. It is not remarkable that curves so related could be found by constructing their equations algebraically, but it is worthy of notice that such sets of curves naturally arise as covariants of rational plane curves. This fact has received no attention in mathematical literature, and it is desirable, therefore, to give several examples of such sets of curves, to outline the conditions which give rise to them, and to indicate some analogous facts for curves of hyperspace.

\section{$\S \mathrm{I}$ a. Genesis of Covariant $R^{k}$ of $R^{n}$.}

Let the $R^{n}$ be written parametrically

$$
x_{0}=(\alpha t)^{n}, \quad x_{1}=(\beta t)^{n}, \quad x_{2}=(\gamma t)^{n} .
$$

The $r$ th osculant of the $R^{n}$ at a point whose parameter is $t^{\prime}$ is an $R^{n-r}$ whose parametric equations are

$$
\begin{gathered}
x_{0}=(\alpha t)^{n-r}\left(\alpha t^{\prime}\right)^{r}, \quad x_{1}=(\beta t)^{n-r}\left(\beta t^{\prime}\right)^{r}, \\
x_{2}=(\gamma t)^{n-r}\left(\gamma t^{\prime}\right)^{r} .
\end{gathered}
$$

Suppose the $R^{n-r}$ has a covariant line whose equation is

$$
k_{0} x_{0}+k_{1} x_{1}+k_{2} x_{2}=0,
$$

in which the $k$ 's are functions of degree $p^{\prime}$ in the coefficients of $t$ which occur in the parametric equations of the $R^{n-r}$. If (3) is actually calculated for (2) and $t^{\prime}$ made equal to $t$ (to signify that it has become variable) the result will be a binary $p$-ic in $t\left(p=r p^{\prime}\right)$ which may be written

$$
\left(\alpha^{\prime} t\right)^{p} x_{0}+\left(\beta^{\prime} t\right)^{p} x_{1}+\left(\gamma^{\prime} t\right)^{p} x_{2}=0 .
$$

The envelope of the line (4) is a rational curve of class $p$ whose parametric equations are

$$
\zeta_{0}=\left(\alpha^{\prime} t\right)^{p}, \quad \zeta_{1}=\left(\beta^{\prime} t\right)^{p}, \quad \zeta_{2}=\left(\gamma^{\prime} t\right)^{p} .
$$


This curve is a covariant rational curve of the $R^{n}$ and its point equations may be found from (5).

\section{$\S \mathrm{I} b$. The Rôle of Polars.}

The line section of (1) by a line

$$
\zeta_{0} x_{0}+\zeta_{1} x_{1}+\zeta_{2} x_{2}=0
$$

may be written symbolically

$$
(a \zeta)(\pi t)^{n}=0
$$

and the binary $(2 n-2)$-ic whose roots are the parameters of the $2 n-2$ tangents from a point (6) may be similarly written

$$
(A x)(\delta t)^{2 n-2}=0 .
$$

There are sets of parameters on the $R^{n}$ which are covariant; i. e., the binary form of which they are roots is unaltered by a linear transformation of the $x$ 's or $t$ 's. For instance, the parameters of the $3 n-6$ flexes, or the $(n-1)(n-2)$ nodal parameters, as well as the set of parameters cut out of $R^{n}$ by any covariant locus constitute such a set. Suppose such a set is given by

$$
(c t)^{q}=0 .
$$

The polar of (9) with respect to (7) or of (7) with respect to (9) according as $q>n$ or $<n$ yields a covariant rational point curve of $R^{n}$ of degree $q-n$ or $n-q$. If $n=q$, the apolarity condition of (7) and (9) is the equation of a covariant point of the $R^{n}$. Similarly the polar of (8) with respect to (9) or of (9) with respect to (8) is the equation of a covariant rational line curve of degree $2 n-2-q$ or $q-2 n+2$, according as $2 n-2>q$ or $<q$. If $2 n-2=q$, the apolarity condition is the equation of a covariant line of the $R^{n}$. Several illustrations will bring out these facts more clearly.

§II a. A Set of Collinear Conics.

Let the $R^{4}$ be written

$$
x_{i}=a_{i} t^{4}+b_{i} t^{3}+c_{i} t^{2}+d_{i} t+e_{i} \quad(i=0,1,2) .
$$

The parameters of its six flexes* are the roots of

* J. E. Rowe, "Important covariant curves and a complete system of invariants of the rational quartic curve," Transactions, vol. 12 (July, 1911), p. 299. 


$$
\begin{aligned}
\alpha^{\prime} t^{6}+3 \lambda^{\prime} t^{5}+\left(3 \beta^{\prime}+6 n^{\prime}\right) t^{4}+(\gamma+8 \delta) t^{3}+(3 \beta+6 n) t^{2} & \\
& +3 \lambda t+\alpha=0 .
\end{aligned}
$$

The line section of 10 by the line (6) is

$$
(a \zeta) t^{4}+(b \zeta) t^{3}+(c \zeta) t^{2}+(d \zeta) t+(e \zeta)=0
$$

the polar quadratic of (12) with respect to (11) multiplied by 20 is

$$
\begin{gathered}
L_{\rho}=\left[4(a \zeta)(\beta+2 n)-(b \zeta)(\gamma+8 \delta)+4(c \zeta)\left(\beta^{\prime}+2 n^{\prime}\right)\right. \\
\left.-10(d \zeta) \lambda^{\prime}+20(e \zeta) \alpha^{\prime}\right] t^{2} \\
+[20(a \zeta) \lambda-8(b \zeta)(\beta+2 n)+2(c \zeta)(\gamma+8 \delta) \\
\left.-8(d \zeta)\left(\beta^{\prime}+2 n^{\prime}\right)+20(e \zeta) \lambda^{\prime}\right] t \\
+[20(a \zeta) \alpha-10(b \zeta) \lambda+4(c \zeta)(\beta+2 n) \\
\left.-(d \zeta)(\gamma+8 \delta)+4(e \zeta)\left(\beta^{\prime}+2 n^{\prime}\right)\right]=0
\end{gathered}
$$

This is a line section of the conic* whose parametric equations are

$$
\begin{array}{rlr}
x_{i} & =\left[4 a_{i}(\beta+2 n)-b_{i}(\gamma+8 \delta)+4 c_{i}\left(\beta^{\prime}+2 n^{\prime}\right)-10 \delta_{i} \lambda^{\prime}\right. \\
& & \left.+20 e_{i} \alpha^{\prime}\right] t^{2} \\
& +\left[20 a_{i} \lambda-8 b_{i}(\beta+2 n)+. . .\right] t & \\
& +\left[20 a_{i} \alpha-10 b_{i} \lambda+. . .\right] & (i=0,1,2) .
\end{array}
$$

A line cuts the $R^{4}$ and (14) in six points whose parameters are apolar to (11).

The parameters of a covariant pair of points on the $R^{4}$ are given by the quadratic

$$
\left(\beta^{\prime}-3 n^{\prime}\right) t^{2}+(\gamma-2 \delta) t+(\beta-3 n)=0 .
$$

The polar quadratic of (15) with respect to (12), multiplied by 12 , may be written in the form

$$
\begin{aligned}
L_{s} & =\left[2(c \zeta)\left(\beta^{\prime}-3 n^{\prime}\right)+3(b \zeta)(2 \delta-\gamma)+12(a \zeta)(\beta-3 n)\right] t^{2} \\
& +\left[6(d \zeta)\left(\beta^{\prime}-3 n^{\prime}\right)+4(c \zeta)(2 \delta-\gamma)+6(b \zeta)(\beta-3 n)\right] t \\
& \left.+12(e \zeta)\left(\beta^{\prime}-3 n^{\prime}\right)+3(d \zeta)(2 \delta-\gamma)+2(c \zeta)(\beta-3 n)\right]=0 .
\end{aligned}
$$

A line section of (16) together with (15) form a binary quartic

\footnotetext{
* We call the discriminant of (13) $P$; the same function of (16) $S$.
} 
which is apolar to the binary quartic defining collinear points of $R^{4}$.

The flex lines of all first osculants of the $R^{4}$ envelope a

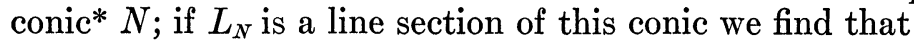

$$
2\left(L_{\rho}-2 L_{s}\right)=5 L_{N} \text {. }
$$

Also, the flex tangents of all first osculants form triangles whose vertices are on a conic $\dagger B$; if $L_{B}$ is a line section of this conic,

$$
9 L_{\rho}+2 L_{s}=2 L_{B} \text {. }
$$

Of these four conics (14), (16), and $B$ may be found by the method described in $\S \mathrm{I} b$; the conic $N$ may be derived as the curves in $\S \mathrm{I} a$.

For convenience in writing let the equations of $P$ in (14) be written

$$
x_{i}=P_{0 i} t^{2}+P_{1 i} t+P_{2 i},
$$

and those of $S$, which may be read off from (16), be put in the form

$$
x_{i}=s_{0 i} t^{2}+s_{1 i} t+s_{2 i} .
$$

By reason of (17), (18), (19) and (20) the parametric equations of $N$ and $B$ are

$$
\begin{gathered}
x_{i}=\left(P_{0 i}-2 s_{0 i}\right) t^{2}+\left(P_{1 i}-2 s_{1 i}\right) t+\left(P_{2 i}-2 s_{2 i}\right), \\
x_{i}=\left(9 P_{0 i}+2 s_{0 i}\right) t^{2}+\left(9 P_{1 i}+2 s_{1 i}\right) t+\left(9 P_{2 i}+2 s_{2 i}\right) \\
(i=0,1,2 .)
\end{gathered}
$$

Suppose the coordinates of the points whose parameter on each of these is $t_{1}$ are found by substituting $t_{1}=t$ in equations (19)-(22). If the coordinates of this point $t_{1}$ on (19) are $\pi_{i}$, and those of the point $t_{1}$ on (20) are $\Sigma_{i}$, the coordinates of the points on $N$ and $B$ with this same parameter value are $\pi_{i}-2 \Sigma_{i}$ and $9 \pi_{i}+2 \Sigma_{i}$. Hence, the points which have the same parameter value on the four conics $P, S, N$ and $B$ are collinear. Evidently this relation is true for all conics whose line sections are of the form

$$
a L_{\rho}+b L_{s}+c L_{N}=0 .
$$

${ }^{*} \mathrm{~W}$. Stahl, "Ueber die rationale ebene Curve vierter Ordnung," Crelle, vol. 101 (1887), p. 314.

† Loc. cit., p. 306. 


\section{$\S$ II $b . \quad A$ Set of Collinear Line Curves.}

As a second illustration we may take a set of covariant rational line curves of the $R^{5}$ which introduce an interesting new feature.

In (1), let $n=5$. The first osculant of $R^{5}$ at $t^{\prime}$ is

$$
x_{0}=(\alpha t)^{4}\left(\alpha t^{\prime}\right), \quad x_{1}=(\beta t)^{4}\left(\beta t^{\prime}\right), \quad x_{2}=(\gamma t)^{4}\left(\gamma t^{\prime}\right) .
$$

The $R^{4}$ possesses a pencil of covariant lines whose properties have been discussed in a previous paper.* These lines are of degree eleven in the coefficients of the equations of the $R^{4}$. If this pencil of lines is

$$
\lambda_{1} L_{3}+\mu_{1} L_{4}=0
$$

and these are actually calculated for (24), the result is a system of rational curves of class eleven such that the tangents to any three at a point whose parameter is given are concurrent lines. The proof of this fact is exactly similar to the proof of the theorem in the last section, both being a matter of linear dependence. In fact, all rational curves of class eleven formed in this way by assigning particular values to $\mu_{1} / \lambda_{1}$ are related in the same manner.

In particular if $\lambda_{1}=-1$ and $\mu_{1}=5$ in (25), the result is not another line whose coefficients are of degree eleven in the coefficients of the parametric equations of the $R^{4}$, but the product of an invariant of the $R^{4}$ of order six in these coefficients and a line $L_{1}$ whose coefficients are of degree five in the coefficients of the $R^{4}$. The invariant just mentioned calculated for (24) yields a binary sextic which equated to zero has roots which are a set of covariant parameters on the $R^{5}$. The line $L_{1}$ for (24) envelopes a rational curve of class 5 . Hence, if a particular value of $t$ is substituted in the equations of any two eleventhics derived from (25) and in the equations of the rational quintic just mentioned, the results are the coordinates of three concurrent lines.

Similarly, the $R^{n}$ could have three covariant rational line or point curves, all of different degrees, collinearly related. If $C_{a}, C_{\beta}, C_{\gamma}$ are three line sections or point projections of three covariant rational point or line curves of $R^{n}$, then this set of covariant curves of $R^{n}$ is in collinear relation if only a relation of the form

$$
\lambda I_{1} C_{\alpha}+\mu I_{2} C_{\beta}+\nu I_{3} C_{\gamma}=0
$$

* Transactions, vol. 13 (1912), pp. 388-389. 
exists, where $\lambda, \mu$, and $\nu$ are constants and $I_{1}, I_{2}$, and $I_{3}$ are invariants of the $R^{n}$.

\section{§ III. Extension to Space.}

The processes which have been outlined and illustrated for rational plane curves can be extended directly to space of higher dimensions. Let the $R_{m}{ }^{n}$ denote a rational curve of order $n$ in space of $m$ dimensions. A covariant linear form of an $R_{m}{ }^{n-r}$ in $x$ or $\zeta$, by the use of osculants, gives rise to a covariant rational class or order curve of the $R_{m}{ }^{n}$. The existence of covariant sets of parameters on $R_{m}{ }^{n}$ and the use of polars makes possible the derivation of other covariant rational curves of the $R_{m}{ }^{n}$. The criterion for the existence of collinear curves, as in the case of plane curves, is merely a matter of linear dependence but the number of varieties of collinearity will increase with $m$. For instance, two varieties of collinearity are possible when $m=3$ and these may be illustrated as follows: First, let the $R_{3}{ }^{n}$ have a set of covariant rational curves whose parametric point* equations are given, and let $A, B, C$, and $D$ be the sections of four curves of the set by a plane; if a relation of the form

$$
\lambda A+\mu B+\nu C=D
$$

exist, in which $\lambda, \mu$, and $\nu$ are constants, a parameter substituted in the parametric point equations of these four curves yields the coordinates of four coplanar points. Second, let the $R_{3}{ }^{n}$ have a set of covariant rational curves whose parametric point equations are known, and let $A^{\prime}, B^{\prime}$, and $C^{\prime}$ be the sections of three of these curves by a plane; if a relation of the form

$$
\lambda^{\prime} A^{\prime}+\mu^{\prime} B^{\prime}=C^{\prime}
$$

exist, in which $\lambda^{\prime}$ and $\mu^{\prime}$ are constants, any parameter substituted in the parametric point equations of these curves yields the coordinates of three points on a line.

Dartmouth College, November, 1912.

* I have inserted the word "point" because it is desirable to emphasize the fact that these curves are to be considered loci of a point. 